\title{
Systematic module approach identifies altered genes and pathways in four types of ovarian cancer
}

\author{
JING LIU ${ }^{1}$, HUI-LING WANG ${ }^{2}$, FENG-MEI MA ${ }^{3}$, HONG-PING GUO ${ }^{1}$, \\ NING-NING FANG ${ }^{4}$, SHAN-SHAN WANG ${ }^{5}$ and XIN-HONG LI ${ }^{6}$ \\ ${ }^{1}$ Physical Examination Center; ${ }^{2}$ Department of Gynecology; ${ }^{3}$ Department of Infectious Disease; ${ }^{4}$ Intensive Care Unit; \\ ${ }^{5}$ Department of Obstetrics, People's Hospital of Binzhou, Binzhou, Shandong 256610; ${ }^{6}$ Department of Internal Medicine, \\ Jinan Central Hospital, Jinan, Shandong 250013, P.R. China
}

Received January 14, 2016; Accepted January 9, 2017

DOI: $10.3892 / \mathrm{mmr} .2017 .7649$

\begin{abstract}
The present study aimed to identify altered genes and pathways associated with four histotypes of ovarian cancer, according to the systematic tracking of dysregulated modules of reweighted protein-protein interaction (PPI) networks. Firstly, the PPI network and gene expression data were initially integrated to infer and reweight normal ovarian and four types of ovarian cancer (endometrioid, serous, mucinous and clear cell carcinoma) PPI networks based on Spearman's correlation coefficient. Secondly, modules in the PPI network were mined using a clique-merging algorithm and the differential modules were identified through maximum weight bipartite matching. Finally, the gene compositions in the altered modules were analyzed, and pathway functional enrichment analyses for disrupted module genes were performed. In five conditional-specific networks, universal alterations in gene correlations were revealed, which leads to the differential correlation density among disrupted module pairs. The analyses revealed 28, 133, 139 and 33 altered modules in endometrioid, serous, mucinous and clear cell carcinoma, respectively. Gene composition analyses of the disrupted modules revealed five common genes (mitogen-activated protein kinase 1, phosphoinositide 3-kinase-encoding catalytic 110-KD $\alpha$, AKT serine/threonine kinase 1, cyclin D1 and tumor protein P53) across the four subtypes of ovarian cancer. In addition, pathway enrichment analysis confirmed one common pathway (pathways in cancer), in the four histotypes. This systematic module approach successfully identified altered genes and pathways in the four types of ovarian cancer. The extensive differences of gene correlations result in dysfunctional modules, and the
\end{abstract}

Correspondence to: Dr Xin-Hong Li, Department of Internal Medicine, Jinan Central Hospital, 105 Jiefang Road, Jinan, Shandong 250013, P.R. China

E-mail: lixinhong389@163.com

Key words: ovarian cancer, protein-protein interaction network, module, pathway coordinated disruption of these modules contributes to the development and progression of ovarian cancer.

\section{Introduction}

Among women, ovarian cancer has a high mortality rate and is the fifth leading cause of cancer-associated mortality, behind cancer of the lung and bronchus, and colorectal, breast and pancreatic cancer (1). The average age of ovarian cancer onset occurs later in reproductive life (2). The disease can advance rapidly with transcoelomic spreading from the ovary to other organs and peritoneal surfaces, and with ascites accumulation (3). Of cases of primary ovarian cancer, $\sim 90 \%$ are epithelial carcinoma from the ovarian surface epithelium $(4,5)$. Ovarian epithelial cancer predominantly contains four histotypes of epithelial tumor, including endometrioid, serous, mucinous and clear cell carcinoma. The serous type is the predominant form in women (3). It is well known that early-stage ovarian cancer (stage I/II) is difficult to diagnose, as it is frequently asymptomatic. Therefore, the majority of patients suffering from ovarian cancer are in advanced stages (III and IV) at the time of the initial diagnosis $(6,7)$. To date, the treatment of ovarian cancer is primarily via platinum-based chemotherapy, debulking surgery and radiotherapy, however, the five-year survival rate has only improved only marginally in the last 40 years, remaining $<40 \%$ (8). Thus, it is critical that effective and sensitive diagnostic biomarkers are examined, which can be applied in the early stage of ovarian cancer and improve survival rates of patients.

Ovarian carcinogenesis is caused by accumulated genetic or genomic alterations (9). DNA-microarray technology enables examination of the expression of thousands of genes simultaneously in tumor samples. Data-analysis software, a high-throughput technology, has made it possible to distinguish gene expression profiling between normal and cancer samples, and thus identify differentially expressed genes during cancer development and progression (10). Gene expression profiling can provide information for the mining of novel biomarkers. Based on oligonulceotide arrays, 275 genes have been predicted with increased/decreased expression in ovarian cancer (11). Several characteristic biomarkers involved in ovarian cancer have been determined, including 
E-cadherin (12), carbohydrate antigen-125 (13), cytochrome P450 1B1 (14), cyclooxygenase 1 (15), AKT serine/threonine kinase 2 (16), BRCA (17) and the human epidermal growth factor receptor family $(16,18)$. The dual detection of hepatocyte nuclear factor- $1 \beta$ and napsin $A$ have been reported to be sensitive markers for diagnosing ovarian clear cell carcinoma, which may also be useful for distinguishing ovarian clear cell carcinoma from endometrioid, serous carcinoma and metastatic Krukenberg tumors (19). However, the compensatory mechanisms in four histotypes of ovarian cancer remain to be fully elucidated, and the underlying diagnostic and therapeutic targets require further investigation.

It is known that protein complexes are key molecular entities and they integrate multiple gene products to perform cellular functions (20). Based on advances in high-throughput analysis technologies, substantial protein-protein interaction (PPI) data has been excavated, therefore, it is possible to investigate protein functions systematically (21). However, due to the technological limitations and dynamic nature of protein interaction maps, the protein interaction data produced by high-throughput experiments often possess high false positive and false negative rates, which lead to difficulties in predicting protein complexes accurately (22). Therefore, a systematic method is required to track gene and module behavior across diseases conditions in a controlled manner (23).

The present study aimed to further elucidate the mechanisms of four histotypes of ovarian cancer, therefore, the disrupted modules from reweighted PPI networks were tracked to systematically identify dysfunctional genes and pathways in samples of the four histotypes of ovarian cancer. Initially, based on Spearman's correlation coefficient (SCC) of gene interactions, normal and disease-specific PPI networks were inferred. Subsequently, the clique-merging algorithm was used to examine modules in the re-weighted PPI networks, and the modules obtained in cancer were compared with those in the normal condition to determine altered modules. Finally, the associated functional pathways of the different histotypes were identified, based on the Kyoto Encyclopedia of Genes and Genomes (KEGG) database.

\section{Materials and methods}

Affymetrix chip data. The EMBL-EBI (http://www.ebi.ac.uk/) database provides freely available data from life science experiments, performs basic investigations in computational biology and offers a training program to extensive users. The E-GEOD-6008 dataset, which included 99 individual ovarian tumor samples (37 endometrioid, 41 serous, 13 mucinous and eight clear cell carcinomas) and four normal ovarian samples, was downloaded from EMBL-EBI (24). The RNA expression in each sample was analyzed using an Affymetrix GeneChip Human Genome HG-U133A array.

Data preprocessing. The Affy package (v1.48.0) in R (bioconductor.org/packages/release/bioc/html/affy.html) was used for the analysis of oligonucleotide arrays to delete undesirable values (25). The data preprocessing was performed using the robust multichip average method in the affy package, comprising background correction, normalization, perfect match/mismatch matching and expression value aggregate calculation (25). Subsequently, the raw data in the CEL files were converted to probe-level data, and the probe-level data were transformed to gene symbols. The FeatureFilter function was applied to discard probes that did not correspond to any gene symbol. A final total of 12,493 genes were obtained.

PPI network construction. As is already known, proteins rarely exert their functions individually, however, they are important in a variety of biological process in the form of large protein functional groups (26). Therefore, in the present study, the PPIs of the 12,493 genes were analyzed using the online Search Tool for the Retrieval of Interacting Genes (STRING) tool (string-db.org). Cytoscape software (v3.3.0; www.cytoscape.org), a biological graph visualization tool (27), was used to construct the PPI networks. All 1,048,576 interactions datasets were downloaded from the STRING database to construct the PPI networks. Following the elimination of self-loops, a complicated PPI network was constructed, which comprised 9,273 nodes and 58,617 interactions with a combine-score $\geq 0.75$.

PPI network re-weighting. By obtaining intersection elements of the 12,493 genes in E-GEOD-6008 and the 9,273 nodes in the PPI network, a sub-network of 7,264 nodes and 45,286 interactions was obtained. The weights of interactions reflect their reliabilities, and low absolute scores of interactions may indicate false positives (28). In the present study, the SCC, which describes the association between two variables, was used to evaluate the strength of the association between two paired proteins in the PPI networks. The SCC value ranged from -1 to +1 . The sign of the SCC indicates the direction of association between $\mathrm{X}$ (the independent variable) and $\mathrm{Y}$ (the dependent variable). If $\mathrm{Y}$ increases when $\mathrm{X}$ increases, the SCC is positive. If $\mathrm{Y}$ decreases when $\mathrm{X}$ increases, the SCC is negative. A coefficient of -1 indicates that there is a perfect inverse association between $\mathrm{X}$ and $\mathrm{Y}$. A coefficient of +1 demonstrates that there is a perfect positive association between $\mathrm{X}$ and $\mathrm{Y}$. An SCC of 0 indicates that there is no tendency for $\mathrm{Y}$ to either increase or decrease when $\mathrm{X}$ increases. The SCC increases in magnitude as $\mathrm{X}$ and $\mathrm{Y}$ become closer to being perfect monotone functions of each other. When $\mathrm{X}$ and $\mathrm{Y}$ are perfectly monotonically associated, the SCC is 1 .

Spearman's rank formula was used to calculate the coefficient of two paired proteins $\mathrm{X}$ and $\mathrm{Y}$ in the PPI network. The formula was as follows:

$$
R=1-\frac{6 \sum d_{i}^{2}}{n\left(n^{2}-1\right)}
$$

where ' $R$ ' is the coefficient, ' $d$ ' is the difference between the ranks of corresponding values $\mathrm{X}$ and $\mathrm{Y}$, ' $\operatorname{sum}\left(\mathrm{d}^{2}\right)$ ' is the total of the ' $\mathrm{d}^{2}$ ' column, and ' $\mathrm{n}$ ' is the number of observations. In the present study, the SCC of a gene-gene interaction was defined as the weight value of the interaction.

Module identification. Similar to the method described by Liu et al (28), the module-identification algorithm was performed in three steps based on clique-merging. Firstly, all of the maximal cliques from the weighted PPI networks of the normal sample and four histotypes were selected out, respectively. The maximal cliques were enumerated utilizing a fast 
depth-first method with a pruning-based algorithm, described by Tomita et al (29). Subsequently, a score was assigned to each clique, and the clique score $(\mathrm{C})$ was referred to as its weighted density:

$$
\operatorname{score}(C)=\frac{\sum_{u \in C, v \in C} w(u, v)}{|C| \cdot(|C|-1)}
$$

where $\mathrm{w}(\mathrm{u}, \mathrm{v})$ represents the interaction weight between $\mathrm{u}$ and $\mathrm{v}$, based on the fast depth-first method.

In the third step, the cliques were arrayed in descending order based on their weighted density and the highly overlapped cliques were removed in order to reduce the size of the result. The highly overlapped cliques were merged to construct larger, dense sub-graphs. The term 'inter-connectivity' was utilized to confirm whether the two overlapped cliques be merged together or not. The inter-connectivity score $\left(\mathrm{C}_{1}, \mathrm{C}_{2}\right)$ between the non-overlapping proteins of $\mathrm{C}_{1}$ and $\mathrm{C}_{2}$ was computed according to the following formula:

$$
\begin{aligned}
& \text { inter }-\operatorname{score}\left(C_{1}, C_{2}\right) \\
& =\sqrt{\frac{\sum_{u \in\left(C_{1}-C_{2}\right)} \sum_{v \in C_{2}} w(u, v)}{\left|C_{1}-C_{2}\right| \cdot\left|C_{2}\right|} \cdot \frac{\sum_{u \in\left(C_{2}-C_{1}\right)} \sum_{v \in C_{1}} w(u, v)}{\left|C_{2}-C_{1}\right| \cdot\left|C_{1}\right|}}
\end{aligned}
$$

The obtained clique scores were ranked in descending order and denoted as $\left\{\mathrm{C}_{1}, \mathrm{C}_{2}, \ldots, \mathrm{C}_{\mathrm{k}}\right\}$. For every maximal clique $\mathrm{C}_{\mathrm{i}}$ if there existed another maximal clique $C_{j}$, and $C_{j}$ possessed a lower score than $C_{i}$ and $\left|C_{i} \cap C_{j}\right| /\left|C_{j}\right| \geq t_{o}$ (a predefined overlap-threshold), the weighted inter-connecting score was calculated for the distinct nodes between the two cliques. Provided that $C_{j}$ existed, the interconnectivity score $\left(C_{i} C_{j}\right)$ was used as a standard to determine whether to remove $\mathrm{C}_{\mathrm{j}}$ or merge $\mathrm{C}_{\mathrm{j}}$ with $\mathrm{C}_{\mathrm{i}}$. If the inter-score $\left(\mathrm{C}_{\mathrm{i}}, \mathrm{C}_{\mathrm{j}}\right)$ was higher than or equal to the predefined merge-threshold $t_{m}, C_{j}$ and $C_{i}$ were merged to obtain a module; if not, $\mathrm{C}_{\mathrm{j}}$ was removed. In the present study, the overlap-threshold was 0.5 and the merge-threshold was 0.25 .

Differential module identification. Random statistical analysis was performed for the obtained modules in the four subtypes of ovarian cancer. A p-value cutoff of 0.01 can reveal numerous false positive results and requires another factor. False discovery rate (FDR), one of the most widely used multiple testing criterions for controlling errors of false discoveries, was utilized to adjust the P-value obtained via random statistical analysis. The FDR was first defined by Benjamini et al (30) as the expected proportion of the number of falsely rejected hypotheses among the total number of rejected hypotheses. In the present study, modules with an adjusted $\mathrm{P}<0.01$ based on the FDR measure were considered to be disrupted modules.

Pathway enrichment analysis of genes in disrupted modules. The Database for Annotation, Visualization and Integrated Discovery (DAVID; david.ncifcrf.gov) is a comprehensive functional annotation software program, and is used for integrative and systematic analysis of large gene groups (31). In the present study, KEGG (www.genome.jp/kegg) pathway enrichment analysis was performed using DAVID with the threshold of FDR-adjusted $\mathrm{P}<0.001$ for genes from the altered modules of endometrioid, serous, mucinous and clear cell carcinomas samples, respectively. For the enriched pathways, the appearance frequency of every gene was counted. The higher a frequency of a gene, the higher its level of involvement in pathways and the higher its importance.

\section{Results}

Disruptions in the PPI networks of four types of ovarian cancer. A total of 12,493 genes were obtained from normal ovarian sample and four ovarian cancer (endometrioid, serous, mucinous and clear cell carcinomas) samples using a data preprocessing procedure. The normal ovarian and four ovarian cancer PPI networks reflected equal numbers of nodes $(7,264)$ and interactions $(45,286)$. Subsequently, re-weighted PPI networks of the normal ovarian sample and the four stages of disease were examined using the SCC algorithm. In the normal ovarian and four ovarian cancer networks, the numbers of interactions and average scores (weights) were approximately equal; the 45,286 interactions had average scores of 0.083 (normal), 0.090 (endometrioid carcinoma), 0.085 (serous carcinoma), 0.087 (mucinous carcinoma) and 0.070 (clear cell carcinoma). The correlationwise frequency distributions were different across the normal ovarian and four ovarian cancer networks (Fig. 1). When the expression correlations varied between -1.0 and $-0.8,-0.6$ and -0.4 , and 0.6 and 1.0 , the number of interactions in the normal ovarian network was higher, compared with those in the four types of ovarian cancer. When the expression correlations varied between -0.4 and 0.6 , the number of interactions in the normal network was almost lower, compared with those in the four types of ovarian cancer. In addition, the scores of the total 26,651 interactions in the four ovarian cancer networks were lower, compared with that in the normal network, whereas the total numbers $(18,635)$ of interactions were higher in the disease conditions, compared with that in the normal condition.

Disruptions in the four ovarian cancer modules. The disrupted or altered modules from the normal and four ovarian cancer PPI sub-networks were identified based on the clique-merging algorithm. With the node threshold $>5$, a total of 951 modules were obtained under the five conditions. Comparative analysis for normal and disease modules was then performed to further elucidate the disruptions from a module perspective. Notably, as shown in Table I, the total number of modules (951) and average module size (31.83) were the same across the five conditions, which results from the same interactions. Furthermore, the average weighted density of mucinous carcinoma was marginally higher, compared with that of the other three cancer subtypes. The associations between the numbers of modules and weighted correlation density of the modules are shown in Fig. 2. No significant difference was found between the distribution of modules in the normal and disease conditions at the level of the overall correlation distribution based on the Kolmogorov-Smirnov test $(\mathrm{P}>0.05)$.

Identification of differential modules. In the present study, a total of 28, 133, 139 and 33 differential modules (FDR-adjusted $\mathrm{P}<0.01)$ were identified in the endometrioid, serous, mucinous and clear cell carcinoma, respectively. Extracting genes from 
Table I. Properties of the normal ovarian, and endometrioid, serous, mucinous and clear cell carcinoma modules.

Correlation

\begin{tabular}{lccccc} 
Module set & Number of modules & Mean module size & Maximum & Average & Minimum \\
\hline Normal & 951 & 31.83 & 0.543 & 0.067 & -0.021 \\
Endometrioid & 951 & 31.83 & 0.442 & 0.058 & -0.055 \\
Serous & 951 & 31.83 & 0.693 & 0.058 & -0.158 \\
Nucinous & 951 & 31.83 & 0.551 & 0.072 & -0.088 \\
Clear cell & 951 & 31.83 & 0.396 & 0.049 & -0.167 \\
\hline
\end{tabular}

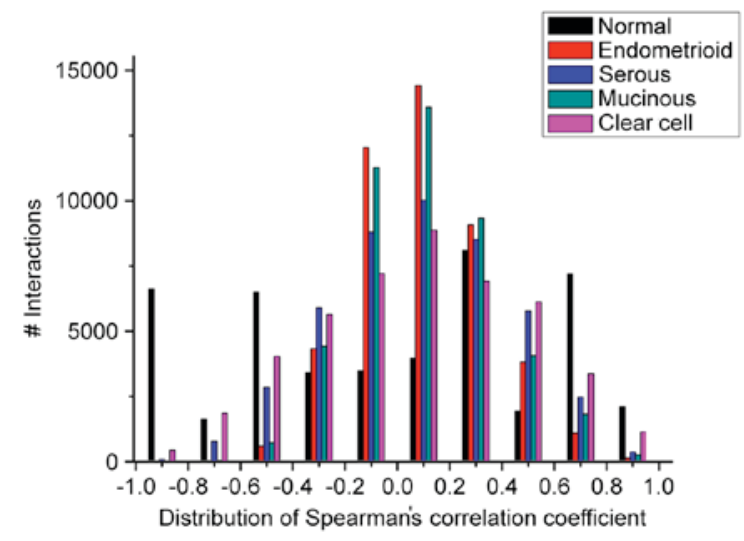

Figure 1. Correlation distribution of interactions in normal ovarian tissue and four types (endometrioid, serous, mucinous and clear cell) of ovarian cancer-specific networks based on Spearman's correlation coefficient.

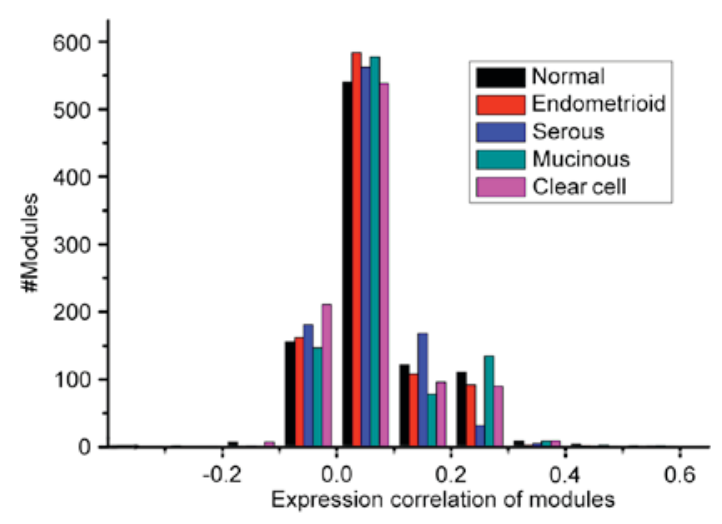

Figure 2. Correlation in distribution of modules in normal and cancerous (endometrioid, serous, mucinous and clear cell carcinoma) ovarian tissues based on module correlation density.

the differential modules in the four types of ovarian cancer resulted in 533, 491, 591 and 408 genes, respectively.

Pathway enrichment analysis of genes in differential modules. DAVID-based KEGG functional pathway analysis was performed for the genes involved in the differential modules. Based on the FDR-adjusted $\mathrm{P}<0.001$, a total of 12, 16, 18 and nine significantly enriched pathways were identified in endometrioid, serous, mucinous, and clear cell carcinoma, respectively. As shown in Fig. 3, pathways in cancer was a common pathway across the four subtypes of ovarian cancer, which may be vital in the development of ovarian cancer. The cell cycle, DNA replication and ribosome pathways were also associated with the subtypes of ovarian cancer, with the exception of endometrioid cancer. In addition, unlike the endometrioid, serous and mucinous carcinoma, clear cell ovarian cancer was associated with several metabolic pathways, including drug metabolism and retinol metabolism. Overall, the cancer-associated pathways were important in the development of ovarian cancer.

By identifying the intersection of the common genes across the four ovarian cancer subtypes and the most frequent genes in the disrupted pathways, five key genes were obtained: Mitogen-activated protein kinase $1(M A P K 1)$, phosphoinositide 3-kinase-encoding catalytic $\alpha$ (PIK3CA), AKT serine/threonine kinase $1(A K T 1)$, cyclin D1 (CCND1) and tumor protein P53 (TP53), which may perform an essential function in the pathogenesis of the four subtypes of ovarian cancer (Table II).

\section{Discussion}

The aim of the present study was to identify dysregulated genes and pathways in four histotypes of ovarian cancer via systematically tracking the dysregulated modules of reweighted PPI networks. The reweighted PPI networks of the normal and four ovarian cancer histotypes were obtained based on the SCC, and the modules in the PPI networks were identified. By comparing the modules of the normal and four ovarian cancer histotypes, 28, 133, 139 and 33 disrupted modules were obtained for endometrioid, serous, mucinous and clear cell carcinoma, respectively. A total of five common genes (MAPK1, PIK3CA, AKT1, CCND1 and TP53) and one common pathway (pathways in cancer) across the four histotypes were examined based on gene composition and pathway enrichment analyses.

The pathways in cancer pathway covers several types of pathway involved in cancer. Chen et al (32) documented that $\mathrm{PI} 3 \mathrm{~K} / \mathrm{AKT} /$ hypoxia-inducible factor- $1 \alpha / \mathrm{CCND} 1$ pathway is vital in follicle-stimulating hormone-driven ovarian cancer cell proliferation. Genistein suppresses the epithelial-mesenchymal transition and migration efficacies of ovarian cancer cells via the estrogen receptor pathway and downregulation of the transforming growth factor- $\beta$ signaling pathway (33). The extracellular-signal-regulated kinase (ERK) and c-Jun $\mathrm{N}$-terminal kinase (JNK) signaling pathways can be regulated by interleukin-33, and promote ovarian cancer growth and metastasis (34). In addition, activation of the mammalian target 


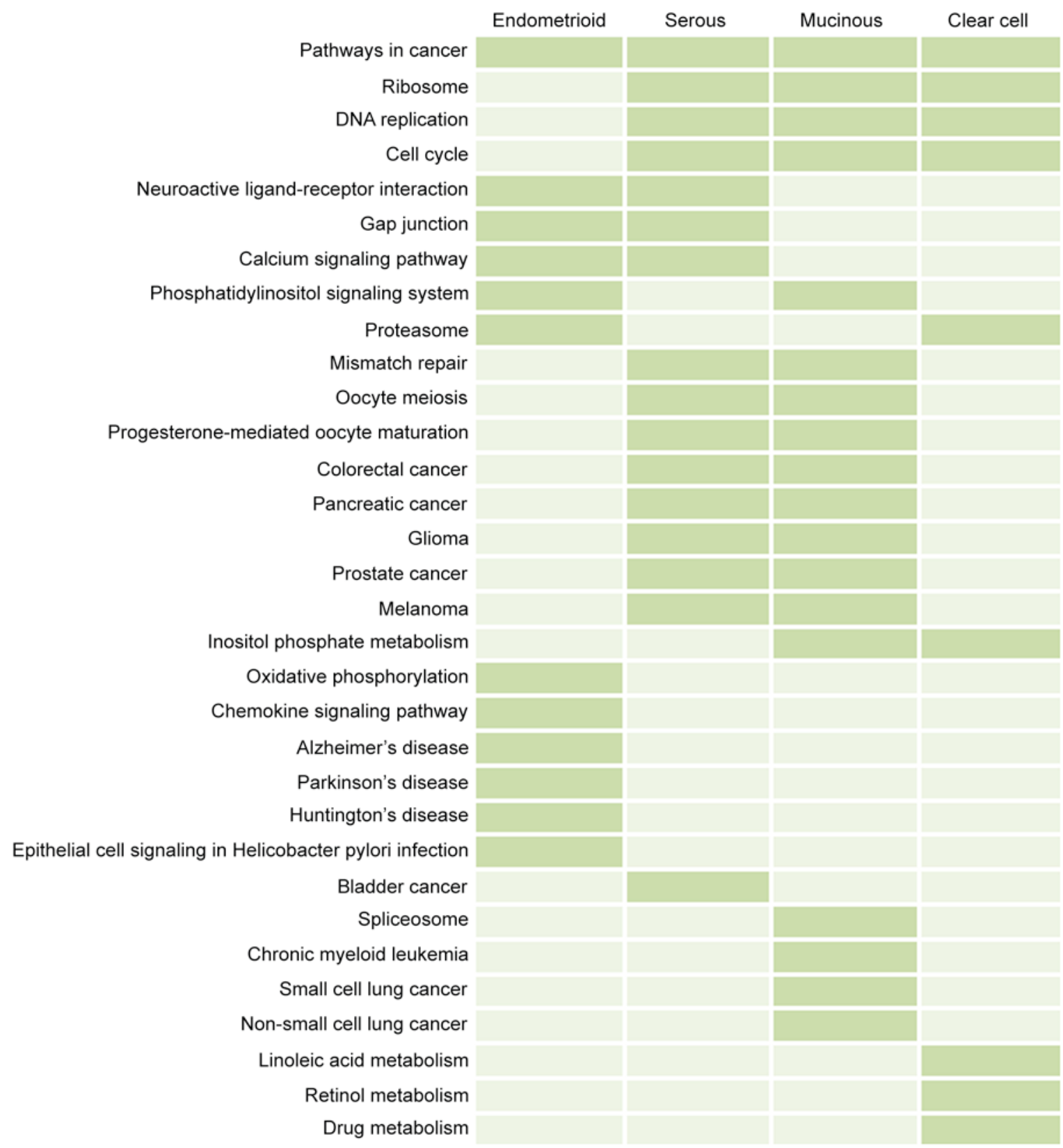

Figure 3. Significant pathways of genes involved in the differential modules in the four types of ovarian cancer (endometrioid, serous, mucinous and clear cell carcinoma) based on $\mathrm{P}<0.001$. Dark green color indicates a present pathway; light green color indicates an absent pathway.

of rapamycin (mTOR) signaling pathway has been demonstrated to promote epithelial ovarian cancer metastasis (35).

MAPKs, a family of serine/threonine protein kinases, including p38 MAPK, ERK1/2, and stress-activated protein kinases (JNK). MAPKs are mediators of comprehensive cellular programs, including cell proliferation, cell differentiation and cell apoptosis, in response to distinct stimuli (36). Studies have found that MAPKs are transiently triggered during mitosis and MAPK activation is involved in the spindle assembly checkpoint (37). Consistently, the protein levels of MAPK1 are increased following demecolcine treatment (38). The proto-oncoprotein, Mos, a serine/threonine kinase, has been recognized as a potent activator of MAPK1 during oocyte maturation $(39,40)$. The overexpression of microRNA-378a-3p or silencing of MAPK1 can reduce the expression level of MAPK1 and enhance adipogenesis (41).
The PIK3CA protein modulates various signals to restrain apoptosis and facilitate cell survival and proliferation in several types of cell $(42,43)$. It has been demonstrated that oncogenic mutations and amplification of PIK3CA can activate the PI3 K/Akt signaling pathway to initiate human papillomavirus-induced tumorigenesis and other types of cancer (44-46). Akt is a serine/threonine protein kinase comprising Akt1, Akt2 and Akt3. Akt1 encodes the principal Akt isoform associated with apoptosis regulation (47). In oropharyngeal cancer, the high protein level of Akt can be an unfavorable prognostic biomarker for relapse-free survival rates in patients (48). The importance of the PI3K/AKT signaling pathway in ovarian cancer has been well documented. In general, this pathway has significant roles in gene transcription, protein synthesis and membrane trafficking, however, the abnormal triggering of this pathway leads to 
Table II. Total 15 genes appearing most frequently in the disrupted pathways of the four types of ovarian carcinoma.

\begin{tabular}{|c|c|c|c|c|c|c|c|}
\hline \multicolumn{2}{|c|}{ Endometrioid } & \multicolumn{2}{|c|}{ Serous } & \multicolumn{2}{|c|}{ Mucinous } & \multicolumn{2}{|c|}{ Clear cell } \\
\hline Gene & Frequency & Gene & Frequency & Gene & Frequency & Gene & Frequency \\
\hline MAPK1 & 20 & MAPK1 & 24 & MAPK1 & 24 & MAPK1 & 20 \\
\hline PIK3CA & 16 & EGFR & 16 & PIK3CD & 20 & PIK3CA & 18 \\
\hline EGFR & 15 & TP53 & 15 & PIK3CA & 20 & AKT1 & 17 \\
\hline PIK3R3 & 15 & CCND1 & 14 & PIK3R2 & 19 & CCND1 & 13 \\
\hline PIK3R2 & 15 & AKT1 & 13 & AKT1 & 18 & TP53 & 12 \\
\hline AKT1 & 14 & PRKCG & 12 & GRB2 & 16 & PRKCG & 11 \\
\hline TP53 & 12 & CDK4 & 11 & CCND1 & 16 & PLCG1 & 11 \\
\hline CCND1 & 12 & EGF & 11 & SOS1 & 16 & PLCG2 & 10 \\
\hline PRKCG & 10 & E2F1 & 10 & EGFR & 15 & RELA & 9 \\
\hline PLCB3 & 10 & E2F3 & 10 & TP53 & 15 & PLCB3 & 9 \\
\hline PLCB1 & 10 & RB1 & 10 & MYC & 12 & NFKB1 & 9 \\
\hline PLCB2 & 10 & MYC & 10 & CDK4 & 11 & CDK4 & 9 \\
\hline PTEN & 9 & PLCB3 & 9 & PLCG1 & 11 & PLCB1 & 9 \\
\hline ERBB2 & 8 & PTEN & 9 & E2F1 & 10 & ADCY9 & 9 \\
\hline MET & 8 & IGF1R & 9 & E2F2 & 10 & PLCB2 & 9 \\
\hline
\end{tabular}

MAPK1, mitogen-activated protein kinase 1; PIK3CA, phosphoinositide 3-kinase-encoding catalytic $\alpha$; AKT1, AKT serine/threonine kinase 1; CCND1, cyclin D1; TP53, tumor protein P53; PLCB1, phospholipase C $\beta 1$; PRKCG, protein kinase C $\gamma$; GRB2, growth factor receptor-bound protein 2; CDK4, cyclin-dependent kinase 4; PLCG, phospholipase C $\gamma$; EGF, epidermal growth factor; SOS1, son of sevenless homolog 1; EGFR, EGF receptor; RB1, retinoblastoma 1; NFKB1, nuclear factor $\kappa B 1$; ERBB2, Erb-B2 receptor tyrosine kinase 2; PTEN, phosphatase and tensin homolog; ADCY9, adenylyl cyclase 9; IGF1R, insulin-like growth factor 1.

cancer initiation, progression and invasion $(49,50)$. PIK3CA and AKT1 amplification are regarded as prognostic factors for ovarian cancer, and the PI3K/AKT/mTOR axis may become a target for drugs.

Another oncogene, CCND1 is a dominating driver of several types of human tumor, including squamous cell and breast cancer, myeloma and Bcell lymphoma $(51,52)$. A previous study indicated that CCND1 was overexpressed in $>50 \%$ of human breast cancer cases (51). In mice, mammary-targeted gene overexpression resulted in mammary tumorigenesis (53). It has been demonstrated that jumonji and AT-rich interaction domain containing 2 can significantly inhibit leukemia cell proliferation by downregulating the expression of CCND1. In addition, the overexpression of CCND1 is closely associated with low-grade ovarian cancer, which is in line with the suggestion that CCND1 is a downstream target for the active MAPK constitutively expressed in ovarian tumors $(54,55)$.

TP53 is a critical transcriptional regulator, which is involved in cell cycle and cell apoptosis upon activation by oncogenes and DNA damage (56). The activated TP53 protein is combined with the regulatory region of target genes to initiate the cell cycle (57). TP53 mutations are frequently screened genetic alterations in ovarian cancer (58). Reles et al (59) reported that the TP53 alteration closely correlates with poor response to chemotherapy, early recurrence and shortened survival rates in patients with ovarian cancer. The high prevalence of TP53 mutations in tubal epithelial carcinoma shows that the TP53 mutations occur in early carcinogenesis. Thus, TP53 mutations are considered to be poor prognostic factors (60). However,
TP53 has been confirmed as an effective blood-based biomarker for the detection of ovarian cancer (61).

In conclusion, the present study successfully identified disrupted modules, including the pathways in cancer module, and hub genes (MAPK1, PIK3CA, AKT1, CCND1 and TP53) in four types of ovarian cancer based on the integrated PPI network. It was inferred that these pathways and genes may be potential biological processes and markers for understanding the mechanism underlying ovarian cancer.

\section{Acknowledgements}

The authors would like to thank the Beijing Springer Medical Research Institute for editing the manuscript.

\section{References}

1. Coleman RL, Monk BJ, Sood AK and Herzog TJ: Latest research and treatment of advanced-stage epithelial ovarian cancer. Nat Rev Clin Oncol 10: 211-224, 2013.

2. Fredrickson TN: Ovarian tumors of the hen. Environ Health Perspect 73: 35-51, 1987.

3. Barua A, Bitterman P, Abramowicz JS, Dirks AL, Bahr JM, Hales DB, Bradaric MJ, Edassery SL, Rotmensch J and Luborsky JL: Histopathology of ovarian tumors in laying hens: A preclinical model of human ovarian cancer. Int J Gynecol Cancer 19: 531-539, 2009.

4. Feeley KM and Wells M: Precursor lesions of ovarian epithelial malignancy. Histopathology 38: 87-95, 2001.

5. Bell DA: Origins and molecular pathology of ovarian cancer. Mod Pathol 18 (Suppl 2): S19-S32, 2005.

6. Moorman PG, Palmieri RT, Akushevich L, Berchuck A and Schildkraut JM: Ovarian cancer risk factors in African-American and white women. Am J Epidemiol 170: 598-606, 2009. 
7. Sharifian A, Pourhoseingholi MA, Norouzinia M and Vahedi M: Ovarian cancer in Iranian women, a trend analysis of mortality and incidence. Asian Pac J Cancer Prev 15: 10787-10790, 2014.

8. Vaughan S, Coward JI, Bast RC Jr, Berchuck A, Berek JS, Brenton JD, Coukos G, Crum CC, Drapkin R, Etemadmoghadam D, et al: Rethinking ovarian cancer: Recommendations for improving outcomes. Nat Rev Cancer 11: 719-725, 2011.

9. Lengauer C, Kinzler KW and Vogelstein B: Genetic instabilities in human cancers. Nature 396: 643-649, 1998.

10. Konstantinopoulos PA, Spentzos D and Cannistra SA Gene-expression profiling in epithelial ovarian cancer. Nat Clin Pract Oncol 5: 577-587, 2008.

11. Meinhold-Heerlein I, Bauerschlag D, Zhou Y, Sapinoso LM, Ching K, Frierson H Jr, Bräutigam K, Sehouli J, Stickeler E, Könsgen D, et al: An integrated clinical-genomics approach identifies a candidate multi-analyte blood test for serous ovarian carcinoma. Clin Cancer Res 13: 458-466, 2007.

12. Ansenberger K, Zhuge Y, Lagman JA, Richards C, Barua A, Bahr JM and Hales DB: E-cadherin expression in ovarian cancer in the laying hen, Gallus domesticus, compared to human ovarian cancer. Gynecol Oncol 113: 362-369, 2009.

13. Jackson E, Anderson K, Ashwell C, Petitte J and Mozdziak PE: CA125 expression in spontaneous ovarian adenocarcinomas from laying hens. Gynecol Oncol 104: 192-198, 2007.

14. Zhuge Y, Lagman JA, Ansenberger K, Mahon CJ, Daikoku T, Dey SK, Bahr JM and Hales DB: CYP1B1 expression in ovarian cancer in the laying hen Gallusdomesticus. Gynecol Oncol 112: $171-178,2009$

15. Zidar N, Odar K, Glavac D, Jerse M, Zupanc T and Stajer D: Cyclooxygenase in normal human tissues-is COX-1 really a constitutive isoform and COX-2 an inducible isoform? J Cell Mol Med 13: 3753-3763, 2009

16. Spandidos DA, Dokianakis DN, Kallergi G and Aggelakis E: Molecular basis of gynecological cancer. Ann NY Acad Sci 900: 56-64, 2000

17. Cancer Genome Atlas Research Network: Integrated genomic analyses of ovarian carcinoma. Nature 474: 609-615, 2011.

18. Lafky JM, Wilken JA, Baron AT and Maihle NJ: Clinical implications of the ErbB/epidermal growth factor (EGF) receptor family and its ligands in ovarian cancer. Biochim Biophys Acta 1785: 232-265, 2008.

19. Li Q, Zeng X, Cheng X, Zhang J, Ji J, Wang J, Xiong K, Qi Q and Huang W: Diagnostic value of dual detection of hepatocyte nuclear factor 1 beta (HNF-1beta) and napsin A for diagnosing ovarian clear cell carcinoma. Int J Clin Exp Pathol 8: 8305-8310, 2015.

20. Gavin AC, Aloy P, Grandi P, Krause R, Boesche M, Marzioch M, Rau C, Jensen LJ, Bastuck S, Dümpelfeld B, et al: Proteome survey reveals modularity of the yeast cell machinery. Nature 440: 631-636, 2006.

21. Jordán F, Nguyen TP and Liu WC: Studying protein-protein interaction networks: A systems view on diseases. Brief Funct Genomics 11: 497-504, 2012.

22. $\mathrm{Wu} \mathrm{C}$, Zhu $\mathrm{J}$ and Zhang $\mathrm{X}$ : Integrating gene expression and protein-protein interaction network to prioritize cancer-associated genes. BMC Bioinformatics 13: 182, 2012.

23. Srihari S and Ragan MA: Systematic tracking of dysregulated modules identifies novel genes in cancer. Bioinformatics 29 : $1553-1561,2013$.

24. Hendrix ND, Wu R, Kuick R, Schwartz DR, Fearon ER and Cho KR: Fibroblast growth factor 9 has oncogenic activity and is a downstream target of Wnt signaling in ovarian endometrioid adenocarcinomas. Cancer Res 66: 1354-1362, 2006.

25. Irizarry RA, Hobbs B, Collin F, Beazer-Barclay YD, Antonellis KJ, Scherf U and Speed TP: Exploration, normalization, and summaries of high density oligonucleotide array probe level data. Biostatistics 4: 249-264, 2003.

26. Nguyen PV, Srihari S and Leong HW: Identifying conserved protein complexes between species by constructing interolog networks. BMC Bioinformatics 14 (Suppl 16): S8, 2013.

27. Shannon P, Markiel A, Ozier O, Baliga NS, Wang JT, Ramage D, Amin N, Schwikowski B and Ideker T: Cytoscape: A software environment for integrated models of biomolecular interaction networks. Genome Res 13: 2498-2504, 2003

28. Liu G, Wong L and Chua HN: Complex discovery from weighted PPI networks. Bioinformatics 25: 1891-1897, 2009.

29. Tomita E, Tanaka A and Takahashi H: The worst-case time complexity for generating all maximal cliques and computational experiments. Theoret Comput Sci 363: 28-42, 2006.
30. Benjamini Y, Drai D, Elmer G, Kafkafi N and Golani I: Controlling the false discovery rate in behavior genetics research. Behav Brain Res 125: 279-284, 2001.

31. Huang da W, Sherman BT and Lempicki RA: Systematic and integrative analysis of large gene lists using DAVID bioinformatics resources. Nat Protoc 4: 44-57, 2009.

32. Chen J, Bai M, Ning C, Xie B, Zhang J, Liao H, Xiong J, Tao X, Yan D, Xi X, et al: Gankyrin facilitates follicle-stimulating hormone-driven ovarian cancer cell proliferation through the PI3K/AKT/HIF-1alpha/cyclin D1 pathway. Oncogene 35: 2506-2517, 2016

33. Kim YS, Choi KC and Hwang KA: Genistein suppressed epithelial-mesenchymal transition and migration efficacies of BG-1 ovarian cancer cells activated by estrogenic chemicals via estrogen receptor pathway and downregulation of TGF- $\beta$ signaling pathway. Phytomedicine 22: 993-999, 2015.

34. Tong X, Barbour M, Hou K, Gao C, Cao S, Zheng J, Zhao Y, $\mathrm{Mu}$ R and Jiang HR: Interleukin-33 predicts poor prognosis and promotes ovarian cancer cell growth and metastasis through regulating ERK and JNK signaling pathways. Mol Oncol 10: 113-125, 2016.

35. Wang Q, Tang Y, Yu H, Yin Q, Li M, Shi L, Zhang W, Li D and Li L: CCL18 from tumor-cells promotes epithelial ovarian cancer metastasis via mTOR signaling pathway. Mol Carcinog 55: 1688-1699, 2016.

36. Su YQ, Wigglesworth K, Pendola FL, O'Brien MJ and Eppig JJ: Mitogen-activated protein kinase activity in cumulus cells is essential for gonadotropin-induced oocyte meiotic resumption and cumulus expansion in the mouse. Endocrinology 143: 2221-2232, 2002

37. Guadagno TM and Ferrell JE Jr : Requirement for MAPK activation for normal mitotic progression in Xenopus egg extracts. Science 282: 1312-1315, 1998.

38. Gao Y, Ren J, Zhang L, Zhang Y, Wu X, Jiang H, Xu F, Yuan B, $\mathrm{Yu} X$ and Zhang J: The effects of demecolcine, alone or in combination with sucrose on bovine oocyte protrusion rate, MAPK1 protein level and c-mos gene expression level. Cell Physiol Biochem 34: 1974-1982, 2014.

39. Posada J, Yew N, Ahn NG, Vande Woude GF and Cooper JA: Mos stimulates MAP kinase in Xenopus oocytes and activates a MAP kinase kinase in vitro. Mol Cell Biol 13: 2546-2553, 1993.

40. Shibuya EK and Ruderman JV: Mos induces the in vitro activation of mitogen-activated protein kinases in lysates of frog oocytes and mammalian somatic cells. Mol Biol Cell 4: 781-790, 1993.

41. Huang N, Wang J, Xie W, Lyu Q, Wu J, He J, Qiu W, Xu N and Zhang Y: MiR-378a-3p enhances adipogenesis by targeting mitogen-activated protein kinase 1 . Biochem Biophys Res Commun 457: 37-42, 2015.

42. Yao R and Cooper GM: Requirement for phosphatidylinositol-3 kinase in the prevention of apoptosis by nerve growth factor. Science 267: 2003-2006, 1995.

43. Liang J and Slingerland JM: Multiple roles of the PI3K/PKB (Akt) pathway in cell cycle progression. Cell Cycle 2: 339-345, 2003.

44. Lee CM, Fuhrman CB, Planelles V, Peltier MR, Gaffney DK, Soisson AP, Dodson MK, Tolley HD, Green CL and Zempolich KA: Phosphatidylinositol 3-kinase inhibition by LY294002 radiosensitizes human cervical cancer cell lines. Clin Cancer Res 12: 250-256, 2006.

45. Keysar SB, Astling DP, Anderson RT, Vogler BW, Bowles DW, Morton JJ, Paylor JJ, Glogowska MJ, Le PN, Eagles-Soukup JR, et al: A patient tumor transplant model of squamous cell cancer identifies PI3K inhibitors as candidate therapeutics in defined molecular bins. Mol Oncol 7: 776-790, 2013

46. Bertelsen BI, Steine SJ, Sandvei R, Molven A and Laerum OD Molecular analysis of the PI3K-AKT pathway in uterine cervical neoplasia: Frequent PIK3CA amplification and AKT phosphorylation. Int J Cancer 118: 1877-1883, 2006.

47. Datta SR, Brunet A and Greenberg ME: Cellular survival: A play in three Akts. Genes Dev 13: 2905-2927, 1999

48. Won HS, Jung CK, Chun SH, Kang JH, Kim YS, Sun DI and Kim MS: Difference in expression of EGFR, pAkt, and PTEN between oropharyngeal and oral cavity squamous cell carcinoma. Oral Oncol 48: 985-990, 2012.

49. Chalhoub N and Baker SJ: PTEN and the PI3-kinase pathway in cancer. Annu Rev Pathol 4: 127-150, 2009.

50. Zhang S and Yu D: PI(3)king apart PTEN's role in cancer. Clin Cancer Res 16: 4325-4330, 2010 
51. Arnold A and Papanikolaou A: Cyclin D1 in breast cancer pathogenesis. J Clin Oncol 23: 4215-4224, 2005.

52. Motokura T, Bloom T, Kim HG, Jüppner H, Ruderman JV, Kronenberg HM and Arnold A: A novel cyclin encoded by a bcl1-linked candidate oncogene. Nature 350: 512-515, 1991.

53. Wang TC, Cardiff RD, Zukerberg L, Lees E, Arnold A and Schmidt EV: Mammary hyperplasia and carcinoma in MMTV-cyclin D1 transgenic mice. Nature 369: 669-671, 1994.

54. Worsley SD, Ponder BA and Davies BR: Overexpression of cyclin D1 in epithelial ovarian cancers. Gynecol Oncol 64: 189-195, 1997.

55. Sui L, Tokuda M, Ohno M, Hatase O and Hando T: The concurrent expression of p27(kip1) and cyclin D1 in epithelial ovarian tumors. Gynecol Oncol 73: 202-209, 1999.

56. Vousden KH and Prives C: Blinded by the light: The growing complexity of p53. Cell 137: 413-431, 2009.

57. Vogelstein B, Lane D and Levine AJ: Surfing the p53 network. Nature 408: 307-310, 2000

58. Lynch HT, Casey MJ, Snyder CL, Bewtra C, Lynch JF, Butts M and Godwin AK: Hereditary ovarian carcinoma: Heterogeneity, molecular genetics, pathology, and management. Mol Oncol 3: 97-137, 2009.
59. Reles A, Wen WH, Schmider A, Gee C, Runnebaum IB, Kilian U, Jones LA, El-Naggar A, Minguillon C, Schönborn I, et al: Correlation of p53 mutations with resistance to platinum-based chemotherapy and shortened survival in ovarian cancer. Clin Cancer Res 7: 2984-2997, 2001.

60. Piek JM, van Diest PJ, Zweemer RP, Jansen JW, Poort-Keesom RJ, Menko FH, Gille JJ, Jongsma AP, Pals G, Kenemans P and Verheijen RH: Dysplastic changes in prophylactically removed Fallopian tubes of women predisposed to developing ovarian cancer. J Pathol 195: 451-456, 2001.

61. Lu D, Kuhn E, Bristow RE, Giuntoli RL II, Kjaer SK, Shih IeM and Roden RB: Comparison of candidate serologic markers for type I and type II ovarian cancer. Gynecol Oncol 122: 560-566, 2011. 